\title{
Criminologie
}

\section{La prison et la responsabilité collective}

\section{Bernard Sweenay}

Volume 9, numéro 1-2, 1976

L’emprisonnement au Québec

URI : https://id.erudit.org/iderudit/017055ar

DOI : https://doi.org/10.7202/017055ar

Aller au sommaire du numéro

Éditeur(s)

Les Presses de l'Université de Montréal

ISSN

0316-0041 (imprimé)

1492-1367 (numérique)

Découvrir la revue

Citer ce document

Sweenay, B. (1976). La prison et la responsabilité collective. Criminologie, 9(1-2), 151-162. https://doi.org/10.7202/017055ar d'utilisation que vous pouvez consulter en ligne.

https://apropos.erudit.org/fr/usagers/politique-dutilisation/ 


\section{LA PRISON ET \\ LA RESPONSABILITÉ COLLECTIVE}

Bernard Sweenay

Je suis né à Montréal, dans le quartier Centre-Sud, le 7 Octobre 1929, dans une famille où nous étions cinq, un père et une mère, au total : sept bouches à nourrir.

J'ai rencontré pour la première fois un juge en 1939; ce fut alors un magistrat de l'enfance.

$\AA$ treize ans, je quittais l'école pour l'école de la vie...

À seize ans, je m'évadais d'une école de protection et, repris, je comparaissais devant la Cour du bien-être social de Montréal pour quitter la salle d'audience avec une condamnation à deux ans et demi à la prison de Bordeaux. Ensuite ce fut une longue période de désespoir... prison?

Un enfant ou un adolescent peuvent-ils mériter d'aller en

Le fait d'enfermer un enfant en «cage» peut-il être utile en quoi que ce soit, à lui et à la société?

Non! C'est inconcevable de mettre un enfant dans une cellule et cela pour plusieurs raisons.

Enfermer un enfant en prison, automatiquement ça forme des futurs criminels, c'est contraire aux lois humaines, c'est contraire à toute la philosophie d'une société bien ordonnée. C'est étrange, on sait comment faire des soldats, on sait comment faire fonctionner une société capitaliste, on a appris à fabriquer des bateaux et des 
avions, on est parvenu même à aller sur la lune, mais on ne sait pas comment arriver à créer une collectivité où il n'y aurait plus de criminels. Ce qui est certain c'est que dans une société comme la nôtre, c'est avec les enfants qu'on fait des criminels. Car, il n'y a pas d'enfants criminels, il y a seulement les adultes qui font des enfants des criminels.

Il y a à peu près deux mois, je suis allé à la Cour du bien-être social. J'ai vu là un petit gars de 8 à 9 ans, dans une cellule. Il y était parce que sa mère l'avait complètement abandonné. Pendant la nuit, les policiers l'avaient emmené à St-Vallier, on l'avait mis dans une cage avec un gros gardien dans la salle attenante, qui pesait à peu près 240 livres... Vous voyez l'image; une cellule, des barreaux à la place de la porte, le néon allumé au plafond et le gros gardien assis derrière son bureau.

Qu'est-ce que ça va refléter dans l'esprit du petit gars qui n'a rien fait de mal...?

Le seule réponse qu'ils m'ont donnée à cette question, c'est : "qu'est-ce que tu veux que je fasse? Ce n'est pas ma job...»

On parle de criminels, en voilà un petit gars qui va l'être peut-être plus tard.

Et je le dis en connaissance de cause...

Mon expérience personnelle remonte à 1941 et à l'École de réforme. La discipline était très, très sévère, les frères de ce temps-là, je dis bien de ce temps-là, étaient sadiques, à un point tel qu'il y en a eu 7 ou 9 d'entre eux qui ont été condamnés justement à la prison. Et, je ne parle pas à travers mon chapeau. On peut fort bien rouvrir certains dossiers de l'époque... En 1946, j'avais 16 ans, on m'a envoyé à la prison de Bordeaux. À 16 ans, j'ai $\mathrm{vu}$ pendre quatre êtres humains... Certains vont dire : "Comment ça se fait que tu as pu voir une exécution?» On est capable de n'importe quoi en prison...

À 16 ans, je n'étais pas un voleur, je n'étais pas un criminel, j'étais tout simplement un rêveur qui se cherchait dans une société qui ne s'est pas retrouvée. Depuis ça n'a pas changé d'ailleurs, on se cherche tous les jours...

Bordeaux, c'était complètement inhumain et puis ce fut le pénitencier fédéral. J'étais là pour vol, c'était encore inhumain, inconcevable, sadique jusqu'à la dernière limite! 
Pénitencier régional St-Vincent-de-Paul, 1948. J'étais dans une cellule du nord de la Tour, pourquoi? Parce qu'on a fait de moi un criminel.

On forme des criminels dans les grandes villes comme Montréal, les centres-villes, où il y a des rats, des coquerelles, des fonds de cours, aucun sens civique, pas de verdure, pas de parc et rien à manger la plupart du temps sur la table. À 12 ans, c'est dur... Et puis personne pour nous dire que faire au juste...

L'école primaire, secondaire; négatif! Pas bon!

Tous les petits gars qui sont là, sont du même quartier... À 12 ans, ce qu'ils entendent, ce dont on parle toujours autour d'eux c'est : la boisson, la bataille, la guerre... Et aujourd'hui, c'est pareil à cette époque-là; aujourd'hui, ces mêmes petits gars entendent surtout parler de la violence!

On leur dit aussi ne fume pas de marijuana, mais le père se saoule avec du whisky, une bonne Molson ou une "Cinquante".

On se dit non violent, on dit que personne ne doit voler, mais on entend parler de hold-up et de fraudes gouvernementales. En 1951 à Ville Jacques-Cartier, on avait voté un million pour refaire les rues, mais on n'a jamais su où cet argent est passé. On a fait une enquête, mais on n'a pas pu découvrir la vérité...

Qui est honnête et qu'est-ce que c'est que d'être honnête?

Les grèves des éducateurs en 1975 à St-Vallier, à Berthelet et ailleurs. Des petits gars de 7 ans, 11 ans et plus ont été détenus, mais là on peut encore protester. Pour les enfants placés dans des foyers de substitution, la situation est moins claire. Pourtant, à l'Université de Montréal, lors d'un séminaire organisé par le juge Trahan, des médecins ont montré des photos des enfants martyrisés par les parents naturels et par les parents nourriciers.

Qui est honnête?

J'ai passé un quart de siècle en prison, pourquoi?

Est-ce qu'il y en a d'autres qui vont passer leur existence en prison?

Probablement oui.

Mais qui fabrique des criminels? 
C'est une belle question qu'il faut se poser! Ce qui est certain, c'est que la prison n'a jamais été un moyen d'éviter la criminalité, parce que la prison forme le criminel. C'est une école du crime!

Il faudrait créer des centres pour recevoir des jeunes, avec des éducateurs qui sont honnêtes, avec probablement des criminologues qui veulent faire des choses et ne réussissent pas parce que ça ne rentre pas dans le cadre de la justice actuelle. Il y a des enfants qui doivent être protégés contre eux-mêmes, mais ce ne sont pas des criminels.

C'est malhonnête d'envoyer un enfant dans une prison, parce qu'il n'y a personne au monde qui peut dire, qu'est-ce qu'un être humain va faire dans 10 ans. Aucun psychiatre, psychologue, aucun criminologue ne peut prévoir qu'est-ce que l'enfant va faire dans 10 ans. C'est absolument faux de dire qu'on est en mesure de le savoir à l'avance. Certes, il est facile de cataloguer les jeunes et de les envoyer un peu partout, mais c'est ainsi qu'ils deviennent des criminels.

Un exemple concret...

Je suis sorti du pénitencier de Dorchester en 1954. Je ne voulais plus retourner en prison. C'était fini non pas parce qu'ils m'avaient réhabilité, mais parce que j'ai changé. La réhabilitation n'existe pas, on ne peut pas "l'enseigner» à un groupe, cela dépend de l'individu lui-même. Il faut que l'individu veuille s'intégrer dans la société. Laquelle, je ne le sais pas, d'ailleurs...

J'étais à l'époque dans l'armée, je suis rentré sous un faux nom, j'avais réussi à passer, je m'arrangeais très bien, j'étais décidé à y rester. C'était vers le mois de juin 1954. J'étais en permission à Montréal. J'avais rencontré sur la rue St-Laurent un ami, mon ancien camarade de cellule. Il m'avait raconté qu'on l'avait libéré du pénitencier, mais que ses parents ne veulent pas le garder et l'obligent à coucher sur la galerie, avec vue sur fond de cour.

J'ai loué une chambre à mon nom et je lui ai donné les clefs. Malheureusement, il a emmené ses bagages et c'était de la marchandise volée. J'ai comparu sous l'accusation du vol avec effraction et comme je ne voulais pas parler, j'ai été condamné à 5 ans de pénitencier pour rien. C'est ça l'amitié et chacun trouve ses amis dans le milieu dans lequel il est obligé de vivre. L'ami en question est mort en prison. Ils l'ont mis au cachot à la suite d'une punition. Il souffrait de maladie de sang et on ne lui a pas 
donné ses médicaments. Il est mort dans la nuit... Il est mort tout seul dans «le trou». Ils ont dit qu'il était drogué. Il y a déjà plusieurs années de ça, mais je n'ai jamais pu oublier. Les amitiés qui se lient en prison, ne sont pas toujours logiques, mais sont solides...

Former un criminel, à l'âge de 12 ans, c'est très facile, parce qu'on est dans un monde où il faut de l'argent pour vivre. Quand l'éducation n'aide pas, quand l'éducation de base n'est pas bonne, on réussit à croire en des valeurs qui n'en sont pas. On donne à un enfant deux possibilités : d'être un petit nègre du Québec, ou d'être un voleur du Québec. Du groupe qui a vécu avec moi dans le bas de la ville, aucun n'a échappé à la prison. Pas un seul... Il faut faire un grand changement. Il faut beaucoup plus d'éducation populaire, beaucoup plus d'aide. Il faut éduquer les êtres jeunes!

On peut dire aux parents, qu'ils ne sont pas bons; qu'ils n'ont pas donné un bon exemple à leurs enfants. Je suis contre le principe d'empiéter sur la liberté individuelle, mais je suis aussi contre les parents qui mettent des enfants au monde et qui ne sont pas capables de s'en occuper. Je suis contre le fait de prendre un petit gars de 7 ans, et de l'envoyer à St-Vallier, à Berthelet, à Boscoville ou au Mont St-Antoine, parce que dans toutes ces institutions on dit : "Qu'est-ce que vous voulez qu'on fasse; il n'est pas bon..."

Il y a un petit gars; son nom c'est Luc et il a 12 ans. Je l'ai rencontré aux Services aux familles. On a parlé quelques minutes ensemble. Je ne savais pas où il s'en allait, je ne le connaissais pas. Le petit gars m'a sauté au cou, il m'a dit : "Joyeux Noël, papan...

On voulait l'envoyer dans un Centre préventif, parce que ses parents sont fous. Les mêmes parents ont mis 7 enfants au monde!

J'ai parlé à son agent de probation, parce qu'il en a un. Il a 12 ans, mais il a déjà un agent de probation comme un criminel adulte, parce que ses parents sont fous. L'agent de probation travaille à la Cour du bien-être social et je lui ai demandé :

- "Qu'est-ce que vous allez faire du petit Luc?»

- «Quand il aura 16 ans, m'a-t-il répondu, on va l'envoyer au Mont St-Antoine.»

Le garçon est catalogué d'avance; quatre ans dans un centre jusqu'à 16 ans, de 16 ans à 18 ans dans une École de protection 
et tout ça parce que ses parents sont des malades mentaux... Est-ce que c'est honnête?

Luc, m'avait écrit une fois. Il a été placé dans une maison dans les Laurentides. C'est un endroit très beau, mais le petit gars, y a été envoyé. Est-ce qu'on a une liberté à 12 ans, ou bien à 15 ans?

On n'a pas de liberté, on ne l'a pas à 45 ans, alors à plus forte raison à 12 ans.

J'ai parlé en l'espace d'un an avec plus de 300 mineurs. Je les ai interrogés sur les raisons pour lesquelles ils abandonnent l'école. Ils répondent : «on n'apprend rien».

Sur 300 jeunes à qui j'ai parlé, il y en a peut-être 150 qui mentent, mais quand même pas tous!

C'est probable, en somme que nos écoles ne remplissent pas leur rôle et il doit y avoir une raison à cela...

Quand on examine les statistiques américaines, on constate que les meurtriers, ce sont fréquemment des mineurs. On affirme qu'en 1975, la criminalité a augmenté de $10 \%$ à cause de la délinquance juvénile, et cela prouve qu'il y a des lacunes graves, que notre éducation n'est pas conforme aux besoins réels des enfants et des jeunes.

Des lacunes existent aussi au niveau de la prévention. Les travailleurs sociaux ne sont pas assez nombreux. Ils doivent s'occuper de trop de jeunes à la fois. Un travailleur social du Centre Sud, qui prétend s'occuper de 150 cas, ne peut pas assumer son rôle auprès de personne, même pas de sa propre femme.

Il n'y a pas non plus, assez de parcs à Montréal, pas assez de centres civiques, mais il y a toujours trop de parents qui mettent des enfants au monde, et qui ne savent pas comment s'en occuper. La Loi donne dans certains cas, assez de pouvoirs aux travailleurs sociaux, qu'ils fassent donc des vérifications dans les familles, avant et après la naissance de l'enfant. Il ne faut pas empiéter sur les libertés individuelles mais il faut protéger les enfants au lieu d'en faire des criminels.

Mes parents à moi sont morts. Quand j'étais enfant, chez nous on mangeait du pain, on buvait de l'eau et on y ajoutait un peu de sucre pour souper... 
Je travaille dans les milieux désavantagés et j'examine les commandes qu'ón donne dans les épiceries. Le vendredi, on achète trois pains et deux caisses de bière. Les enfants, on les voit dans les rues avec du Pepsi, des "chips», au mieux des tablettes de chocolat.

Les jeunes forment des groupes, comme autrefois, quand moi-même j'avais leur âge. Pourquoi qu'ils font cela. Ils veulent appartenir à quelque chose, ils veulent faire des choses, ils ne savent pas au juste quoi... Autrefois on buvait, maintenant on se drogue et c'est encore pire parce que cela détériore plus vite le cerveau.

En fait, les jeunes rêvent de simplicité et d'honnêteté. Pour certains c'est le retour à la terre. Pour d'autres le voyage sur la lune, mais ils veulent tous trouver la simplicité. Ils veulent qu'on leur explique pourquoi il y a des tabous, pourquoi il y a une société, pourquoi il y a une police, pourquoi il y a des criminologues et des travailleurs sociaux, et tout cela de façon claire.

Ils veulent comprendre des choses, ils veulent faire des choses, ils veulent avancer. Ils ne veulent pas aller se battre et ils ne veulent pas faire la guerre...

Autour d'eux, il y a un trop grand étalage de crimes, autant dans les journaux qu'à la télévision, que partout ailleurs.

Et l'attraction est forte! L'attraction de l'aventure, l'attraction de pouvoir faire des choses contraires à la loi, parce qu'on est protestataire...

Il faut protester dans les temps modernes, surtout en Amérique du Nord. Il faut protester contre des choses qui n'ont pas de sens, et c'est leur manière de protester.

Ils commencent en bas de l'échelle, ils font des petits vols à main armée, ils se font prendre, on compile les dossiers, et à 18 ans, le jeune gars a de 90 à 180 charges contre lui de hold-up et de vols d'autos.

On n'a pas le droit de divulguer le dossier d'un délinquant juvénile, mais ils existent. Les renseignements sont consignés dans les registres de la police. Les jeunes sont catalogués à partir de 12 ans, les parents ne veulent plus s'en occuper, on les place dans une école de redressement, ils sortent, se sauvent, ils se retrouvent dans la rue, ils ne savent pas où aller, ils vont revoir leurs amis. 
Et il faut manger, alors on vole ou on va dans les clubs pour homosexuels. Désormais, ces clubs fonctionnent ouvertement parce que $\mathrm{M}$. Trudeau, notre premier ministre a décriminalisé l'homosexualité entre adultes consentants.

Dans les clubs pour homosexuels on ne vérifie pas de trop près l'âge des clients...

On sait toutes sortes de choses quand on a 13 ou 14 ans; on se fait prendre ensuite, on se fait envoyer dans un centre de redressement, on se sauve et tout recommence. Un jour, on décide qu'un jeune peut aller à Boscoville et c'est une "promotion». Car il faut avoir un certain niveau intellectuel et beaucoup de tristes expériences pour être accepté à Boscoville.

J'ai connu cinq garçons qui attendaient d'être admis à Boscoville et qu'on devait garder ailleurs. Car il n'y a que cent places au total, à Boscoville, dont souvent quarante uniquement sont occupées parce qu'on manque de personnel...

Dans la province de Québec, 1300 prévenus et détenus mineurs sont reçus dans les prisons pour adultes. Dans les pénitenciers fédéraux, par contre, on évalue à 2000 environ le nombre des détenus ayant moins de 18 ans, dont 400 environ pour le Québec. Aux États-Unis, juste de l'autre côté de la frontière, c'est une proportion dix fois plus élevée... C'est ça l'Amérique du Nord!

Ils sont jeunes, tous ces garçons et sept fois sur dix, ils ne devraient pas être en prison. Trois sur dix, au grand maximum, doivent vraiment être isolés et encore...

Un jeune qui n'a jamais été arrêté, qui n'a pas commencé au bas de l'échelle, quand à 16 ans, il arrive en prison, il est traumatisé. Pour ma part, quand à 16 ans je me suis retrouvé à Bordeaux, quand ils ont fermé la grosse porte de fer derrière moi, j'ai tout d'abord pleuré et j'ai eu peur. J'avais peur de tout le monde et je ne parvenais pas à comprendre ce qu'il m'arrivait, ni pourquoi. Après, j’ai tout cassé dans ma cellule, j'ai participé à un genre de «bingo" et j'ai été content de me défouler. J'ai vu ensuite ce que les gardes faisaient quand ils se défoulaient à leur tour...

Qu'est-ce qu'on ressent quand on est un être humain et quand à 16 ans, on commence à comprendre les rouages d'une prison? On a peur, on a des crises de rage et on ne croit plus à rien, 
ni à personne... On se fait aussi des amis!... Le moyen de communication, c'est le châssis, mais on sort aussi de la cellule assez librement si on sait s'organiser. Certains copains ont des relations, d'autres en profitent. C'est une collectivité à part, le pénitencier, un univers en soi avec ses usages propres et ses coutumes. Les jeunes ont leurs héros. Ils rêvent de Che Guevara ou d'un autre, mais au pénitencier, le héros c'est l'homme qui est le plus batailleur, le plus dur, celui qui a fait le vol le plus audacieux. À l'opposé, celui qui n'est pas bon, c'est celui qui a dénoncé un copain ou qu'on soupçonne de vouloir le faire.

Les policiers savent qu'il y en a beaucoup qui parlent bien qu'on le cache généralement, que leurs noms ne sont pas divulgués et qu'on ne les cite pas comme témoins...

Au pénitencier, on se raconte aussi des histoires et on finit par croire qu'elles sont vraies. Les jeunes affirment qu'ils ont cinq ou six "blondes" qui les attendent dans toutes les villes du Canada, qu'ils ont une voiture de l'année et au moins 100000 dollars bien cachés. Ils ont aussi des projets fabuleux, des vols qu'ils vont faire après leur libération. Ils sont tous très occupés à l'avance.

- «Dès que je vais sortir il faudra que je rétablisse mes contacts... Que je prenne mon argent, que je...»

Quand on sort, quand on rencontre ensuite les anciens copains, on se redit les mêmes mensonges.

Cette histoire n'a pas marché et il faut que j'attende. On va "refaire un autre coup en attendant le grand coup", et ainsi de suite...

En fait, imaginer qu'un voleur a de l'argent, c'est faux et c'est illogique. S'il avait de l'argent il ne volerait pas. Je ne pense pas qu'on vole pour le «sport". Dans mon cas, ce fut le goût de l'aventure, puis de la vengeance. Ce n'était pas pour posséder des choses ou de l'argent.

C'est un défi de ne pas se faire reprendre, d'ouvrir un coffre-fort qui a la réputation d'être impossible à ouvrir, mais ce n'est pas un sport... De toute façon, je ne conseille pas aux sportifs de commencer ce jeu-là!

À tous les jeunes qui volent, il faudrait dire qu'ils ne le font pas pour avoir de l'argent, mais pour enrichir le receleur. Un jeune 
de 16 ans, par contre, qui peut avoir un salaire de $\$ 80$ par semaine a la possibilité d'économiser.

S'il va en prison pendant un an, il ne mettra pas $\$ 50$ par semaine de côté. Un vol de $\$ 1000$ rapporte moins en réalité que $10 \%$, même si on réussit à ne pas être pris par la police.

Quant à l'aventure, tout le monde aime l'aventure et on peut la vivre en restant honnête.

On peut aller travailler dans d'autres provinces, dans d'autres pays, mais pour ça il faut travailler quelques mois, chez soi, avoir un peu d'expérience et un peu d'argent. Il faut apprendre à conduire, acheter une petite voiture d'occasion, ou une motocyclette et voyager. Il faudrait dire tout cela aux jeunes et aussi que la plus belle aventure, c'est l'aventure intérieure!

C'est ça qu'on a envie d'expliquer aux jeunes, à tous ceux surtout dont les parents ne s'occupent pas et qui deviennent dans neuf cas sur dix des criminels. Car ils sont marqués dès leur enfance...

En dehors de leurs copains, ils parlent tout le temps de leur père et de leur mère.

- «Si ma mère ne buvait pas, si mon père était à la maison, si..." Les trois jeunes dont je m'occupe en ce moment, parlent continuellement de leur mère. Leur père est mort et leur mère est une lâche.

Le petit gars a quitté l'école et il a fait l'école buissonnière. La mère l'avait emmené tout de suite à la Cour du bien-être social.

Un autre jeune me racontait :

Ma mère nous a tous envoyés à la Cour du bien-être social. Nous étions sept à la maison et nous avons passé devant le juge. Dernièrement, ma petite sœur est rentrée à $1 \mathrm{~h}$ du matin. Ma mère l'avait dénoncée à la police et elle a dit au juge que sa plus jeune fille est folle.

Ils manquent d'affection; c'est ça la source de délinquance juvénile, c'est la base de tout le reste, c'est le grand crime des adultes.

Il faudrait dire à certains pères :

Tu sais, tu vieillis, il y a une nouvelle génération qui pousse, tu ne peux pas te mettre au niveau de l'enfant si tu es tout 
le temps saoul. Pour comprendre l'enfance, il faut avoir un certain respect de soi et ne pas être semblable à un animal.

Il y a des parents qui sont des animaux au sens péjoratif de ce terme. Des animaux, des criminels, qui ne sont jamais punis par la loi. Avant de placer l'enfant on devrait faire passer un test d'intelligence aux parents; là on serait honnête avec l'enfant et avec les parents.

Quelles sont les solutions?

On s'efforce de placer les enfants, mais on n'a pas de centres et tout le monde le sait : les juges, le ministre de la Justice et les travailleurs sociaux. On va donc, semble-t-il, créer des nouveaux centres, mais ça va être quoi au juste et pour quelle catégorie de jeunes?

Qu'est-ce qu'un enfant signifie pour la société? Un danger ou un investissement? Croit-on qu'il va faire des révolutions à 12 ans? De quelle protection s'agit-il? Celle de l'enfant ou celle de la société? Qui est-ce qu'on protège au juste? On dit ces jeunes ce sont des voleurs et c'est faux. On a des cas de protection d'enfants à la Cour du bien-être social. Ce ne sont pas des criminels, ils n'ont pas volé, ils n'ont rien fait. Pourtant, ils vont dans les mêmes institutions que les autres... On héberge des jeunes dans l'Ouest de la ville, il y a un centre à Westmount, il y a le Boys' Club et le Y.M.C.A. Malgré la Loi 63, ça marche très bien du côté anglophone. Ça marche très bien, aussi chez les israélites parce qu'ils croient eux à l'enfance et à l'obligation morale de la protéger. Donc, c'est possible d'élaborer des services communautaires autres que les cours de justice, les postes de police et les prisons.

On sait que ça coûte très cher de garder un jeune en prison ou au pénitencier, on sait que ce sont les contribuables qui paient la note... C'est l'homme de la rue qui fait des enfants et c'est lui aussi qui est parfois incapable de s'en occuper! On critique les juges, les services et les structures, mais on refuse de s'impliquer personnellement.

Nous tous collectivement, sommes-nous autre chose que des petits robots qui produisent des biens, mais également des enfants.

Qui est-ce qui les a élevés ces enfants-là? Ce n'est certainement pas monsieur Choquette, ou monsieur Trudeau, ou monsieur Bourassa, ou quelqu'un d'autre qui va prendre le pouvoir. 
C'est la femme qui a mis l'enfant au monde qui l'élève. C'est elle qui est responsable, parce qu'elle savait d'avance qu'elle n'a ni le courage, ni la volonté, ni l'éducation pour élever cet enfant-là, alors de quel droit l'a-t-elle mis au monde et l'a-t-elle gardé au lieu de le confier tout de suite à l'adoption?

L'enfant qui s'en va en prison n'a pas demandé de naître et n'a pas été aidé à temps. La société permet aux adultes de faire des enfants comme General Motors fait des voitures et ensuite enferme dans les prisons les "unités mal fabriquées"...

Les résultats sont là et aussi le désespoir et le drame des enfants mal aimés; les enfants dont on fait des criminels... 